University of Nebraska - Lincoln

DigitalCommons@University of Nebraska - Lincoln

Norman R. Simon Papers

Research Papers in Physics and Astronomy

4-1968

\title{
On The Pulsational Stability of Blue Supergiants
}

Richard Stothers

Goddard Institute for Space Studies

Norman R. Simon

University of Nebraska - Lincoln, nsimon@unl.edu

Follow this and additional works at: https://digitalcommons.unl.edu/physicssimon

Stothers, Richard and Simon, Norman R., "On The Pulsational Stability of Blue Supergiants" (1968).

Norman R. Simon Papers. 24.

https://digitalcommons.unl.edu/physicssimon/24

This Article is brought to you for free and open access by the Research Papers in Physics and Astronomy at DigitalCommons@University of Nebraska - Lincoln. It has been accepted for inclusion in Norman R. Simon Papers by an authorized administrator of DigitalCommons@University of Nebraska - Lincoln. 
The Astrophysical Journal, Vol 152, April 1968

\title{
ON THE PULSATIONAL STABILITY OF BLUE SUPERGIANTS
}

\author{
RICHARD STOTHERS \\ Institute for Space Studies, Goddard Space Flight Center, NASA, New York \\ AND \\ Norman R. SIMON \\ Yeshiva University, New York \\ Received July 27, 1967 \\ ABSTRACT
}

\begin{abstract}
The brightest blue supergiants represent the most massive evolved stars known. They are believed to be in the core helium-burning phase of evolution. To discuss the question of their stability, model interiors of core helium-burning stars with hydrogen-burning shells have been constructed and tested for pulsational instability $(15-1000 M \odot)$. All models are found to be extremely stable against nuclearenergized pulsations despite the high radiation pressure. Consequently, any star with mass less than the critically stable mass on the main sequence should evolve into the blue-supergiant region without disruption. By combining the theoretical calculations with the available observational data for the Large Magellanic Cloud, a self-consistent set of parameters may be obtained for the Large Cloud as follows: (1) the coincidence of the mass of the most luminous supergiants with the critical mass on the main sequence, (2) the occurrence of this mass at about $60 M \odot,(3)$ a hydrogen abundance of $X=075$ in the young Cloud population, and (4) a distance modulus of $(m-M)_{0}=18.7$. We further verify theoretically the remarkable observed constancy of brightness of the most luminous B supergiants and their stability against disruption or large amounts of mass loss; any other kind of variability is likely to be due to atmospheric phenomena or to binary motion. The mass of the brightest Cepheids in the Magellanic Clouds is about $15 M \odot$. In our Galaxy, the most luminous supergiants known have inferred masses which do not significantly exceed the critical mass, nor is there any certain evidence for supermassiveness among the observed $\mathrm{O}$ stars.
\end{abstract}

\section{INTRODUCTION}

In a theoretical investigation of massive stars on the main sequence, Ledoux (1941) and Schwarzschild and Härm (1959) showed that stars with masses greater than a certain critical mass are extremely unstable toward radial pulsations. This instability is basically a consequence of the growing importance of radiation pressure in large stellar masses. Schwarzschild and Härm went on to show that evolution of the stars during hydrogen burning tends to induce stability: the evolutionary effect of high central condensation outweighs the effect of rising radiation pressure and results in smaller pulsation amplitudes near the central nuclear-energy sources. It is these sources which serve as the energizing mechanism for the instability.

At the end of hydrogen burning in the core, the nuclear-energy source moves out to a surrounding shell. The core contracts, and a fresh supply of energy is drawn from the rapid changes in the gravitational field. Eventually the core becomes sufficiently hot to burn helium via the triple-alpha reaction. Thereupon the stellar structure is stabilized under two sources of nuclear energy: helium burning near the center and hydrogen burning in an outer shell. The relative contribution of the shell to the total luminosity reaches a maximum when the gravitational contraction in the core is initially halted by helium burning.

There arises the new possibility of a shell source of energy sufficiently near the surface to produce pulsational instability. In order to investigate this possibility, we have constructed model interiors of massive stars in the blue-supergiant phase of core helium burning. The chosen masses are 15, 60, 200, and $1000 M \odot$. The two highest masses are heavier than the maximum stable mass on the main sequence, but it is not certain a priori that such stars would be disrupted by pulsational instability. Observational evidence can be adduced to decide on this question. 


\section{STELLAR MODELS}

The hydrogen-burning phase of evolution for the four masses has been computed in two previous papers (Stothers 1965, 1966b). On the basis of the detailed computations of evolutionary tracks for 15.6 $M \odot$ (Sakashita, Ôno, and Hayashi 1959; Hayashi and Cameron 1962) and for $30 M \odot$ (Stothers 1963, 1964, 1966a), the intervening phases of core hydrogen exhaustion and gravitational contraction need not be computed in order to obtain reliable models for the helium-burning phase.

The particular stage of evolution with which we are concerned occurs when the star passes into the region of the blue supergiants. Strictly speaking, the structure here depends on the convective modifications and the amounts of hydrogen and helium depletion which take place at the onset of helium burning when the star is a red supergiant (Stothers and Chin 1968). However, the total luminosity is relatively insensitive to the evolutionary changes during helium burning. Since the luminosity determines the nondimensional structure of the envelope, we need only determine the luminosity and then specify an effective temperature in order to obtain a reliable envelope model (which is of interest here). Therefore, we have constructed a "typical" blue-supergiant model for each mass by simply assuming the distribution of chemical composition which the star would have at the onset of core helium burning.

The general structure in the blue-supergiant phase has been adequately described in earlier papers (Stothers 1966a; Stothers and Chin 1968). The hydrogen-burning shell lies at the bottom of an intermediate zone whose frozen distribution of hydrogen and helium (left behind by the retreating convective core during hydrogen burning) is given adequately by the last core hydrogen-burning model. In reality, part or all of the intermediate zone will have had its composition gradient altered by semiconvective mixing near the main sequence and by full convection (extending downward from the surface) in the red-supergiant region at the onset of core helium burning. For simplicity, the semiconvective and fully convective modifications will be ignored and the intermediate zone treated as unmixed and radiative. The hydrogen-burning shell is approximated by a discontinuity in both chemical composition and luminosity. The mass fraction of the shell $\left(g_{s}\right)$ is located by specifying the hydrogen abundance $X_{s}=0.03$; the structure is rather insensitive to the precise location of the shell. The opacity in massive stars with high surface temperatures may be taken as due only to electron scattering, so long as great accuracy is not required (Stothers and Chin 1968). The other assumptions, notations, and nuclear-energy parameters are the same as in Stothers (1966a).

The results of computations for the stable stellar models are presented in Table 1. At higher masses, the diminishing importance of the hydrogen energy source, as measured by the ratio $L_{\mathrm{H}} / L$, may be noted. In Figure 1, the relevant evolutionary tracks of the different masses are sketched in.

Pulsations of the various models were calculated in the usual quasi-adiabatic approximation. The actual procedure adopted was the same as that in previous work on massive stars where electron scattering is the dominant opacity source and nuclear energy is the destabilizing mechanism (Schwarzschild and Härm 1959; Boury 1963; Boury and Ledoux 1965). The relative pulsation amplitudes are all continuous through the star, except for the luminosity perturbation which has a discontinuity at the hydrogen shell. The net rate of gain or loss of pulsational energy is given by

$$
L_{P}=L_{P N}-L_{P H}-L_{P S}
$$

where $L_{P N}$ is the rate of gain from nuclear sources, $L_{P H}$ is the rate of loss due to heat leakage, and $L_{P S}$ is the rate of loss from acoustical waves running off the surface. The pulsational $e$-folding time is given by the reciprocal of the stability coefficient $K$, where $K=L_{P} / 2 E_{P}$ and $E_{P}$ is the mechanical energy of the pulsations. The notations are the same as in Schwarzschild and Härm (1959). 
TABLE 1

TYPICAL MODELS FOR VERY MASSIVE BLUE SUPERgIANTS IN THE CORE HELIUM-BURNING STAGE OF EVOLUTION*

\begin{tabular}{|c|c|c|c|c|}
\hline & \multicolumn{4}{|c|}{$M / M_{\odot}$} \\
\hline & 15 & 60 & 200 & 1000 \\
\hline $\begin{array}{l}q_{s} \\
\beta_{s} \\
\log T_{s} \\
\log \rho_{s} \\
\log \left(r_{s} / R\right) \\
L_{\mathrm{H}} / L \\
\log (L / L \odot) \\
\log (R / R \odot) \\
\log T_{e} \\
\mathrm{Sp} \quad .\end{array}$ & $\begin{array}{rl}0 & 220 \\
0 & 632 \\
7 & 703 \\
0 & 937 \\
-2 & 305 \\
0 & 885 \\
4 & 906 \\
1 & 820 \\
4 & 080 \\
\text { B5 }\end{array}$ & $\begin{array}{rl}0 & 426 \\
0 & 288 \\
7 & 738 \\
0 & 415 \\
-2 & 381 \\
0 & 457 \\
6 & 077 \\
2 & 375 \\
4 & 095 \\
\text { B3 }\end{array}$ & $\begin{array}{rr}0 & 538 \\
0 & 150 \\
7 & 748 \\
0 & 083 \\
-2 & 613 \\
0 & 233 \\
6 & 777 \\
2 & 921 \\
3 & 997\end{array}$ & $\begin{array}{rr}0 & 591 \\
0 & 067 \\
7 & 751 \\
-0 & 300 \\
-2 & 880 \\
0 & 098 \\
7 & 558 \\
3 & 569 \\
3 & 868\end{array}$ \\
\hline
\end{tabular}

$* X_{e}=070, Z_{e}=003, X_{s}=003, Y_{c}=097$

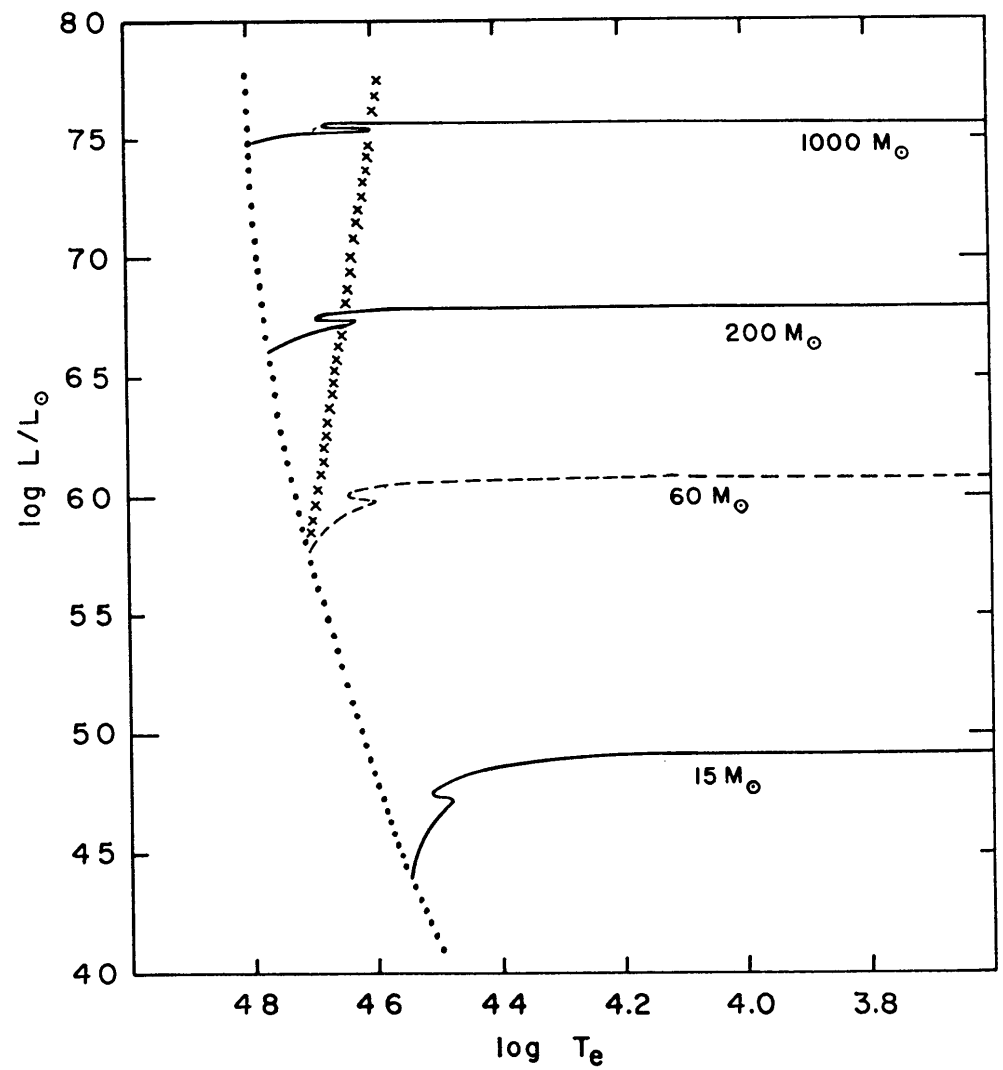

Fig. 1.-Theoretical H-R diagram for very massive stars evolving at constant mass, from the initial main sequence (locus of dots) into later phases of evolution. Stellar models to the left of the line of crosses are theoretically unstable toward radial pulsations. The region of the diagram above the dashed curve (representing the evolutionary track for a star of $60 \mathrm{M} \odot$ ) should be observationally devoid of normal stable stars. 
The pulsational characteristics of the computed models are presented in Table 2. It is clear that these models are pulsationally very stable up to the highest masses. The reason for their stability is the high central condensation, which causes the pulsation amplitudes to drop very rapidly just inside the surface despite the large relative pressure of radiation. Although the hydrogen-burning shell is located halfway out from the center in mass fraction, it is spatially too deep to feed any significant energy into pulsations. This result is similar to the one found for giant-star models of lower mass, where radiation pressure is not important (Cox 1955).

Calculating still higher masses would not change the situation since the mass fraction of the shell (defined where $X=0.03$ ) approaches an asymptotic limit of $q_{s} \sim 0.60$ at very high masses (Stothers 1966b; Stothers and Chin 1968). Nor is the decreased amount

TABLE 2

PUlsational Characteristics OF THE Blue-Supergiant MODELS

\begin{tabular}{|c|c|c|c|c|}
\hline & \multicolumn{4}{|c|}{$M / M \odot$} \\
\hline & 15 & 60 & 200 & 1000 \\
\hline $\begin{array}{l}\omega^{2} \\
\text { Period (days) } \\
\beta_{R} \\
\log \rho_{R} \\
\log g_{R} \\
(\delta r / r)_{R} \\
(\delta T / T)_{R} \\
(\delta L / L)_{R} \\
(\delta r / r)_{s} \\
(\delta T / T)_{s} \\
(\delta L / L)_{s} \\
L_{P N} / L \\
L_{P H} / L \\
L_{P S} / L \\
L_{P} / L \\
1 / K \text { (days) }\end{array}$ & $\begin{array}{rl}8 & 453 \\
5 & 52 \\
0 & 865 \\
-\quad 9979 \\
1 & 975 \\
1 & 000 \\
-\quad 3889 \\
-11554 \\
\sim 000 \\
\sim 000 \\
\sim 000 \\
\sim \quad 000 \\
2623 \\
02074 \\
-2650 \\
1790\end{array}$ & $\begin{array}{rl}6 & 584 \\
21 & 3 \\
0 & 498 \\
-10 & 742 \\
1 & 467 \\
1 & 000 \\
-2 & 748 \\
-6 & 992 \\
\sim 0 & 00 \\
\sim 0000 \\
\sim 0000 \\
\sim 000 \\
\sim 050 \\
11057 \\
0046 \\
-1102 \\
721\end{array}$ & $\begin{array}{rl}5 & 870 \\
81 & 5 \\
0 & 246 \\
-11 & 521 \\
0 & 896 \\
1 & 000 \\
-2 & 485 \\
-5 & 939 \\
\sim 0000 \\
\sim 000 \\
\sim 000 \\
\sim 000 \\
9000 \\
00020 \\
-902 \\
267\end{array}$ & 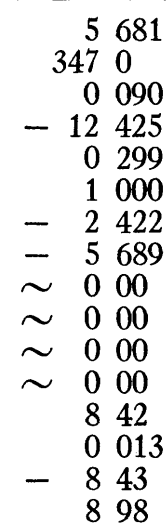 \\
\hline
\end{tabular}

of damping at high masses very likely to offset the increased central condensation. Clearly, the extreme nature of the calculated stability indicates that none of the approximations in our models (including the distribution of hydrogen in the intermediate zone) could qualitatively invalidate our conclusion.

Evolution during core helium burning is accompanied by a burning of the hydrogen shell outward in mass fraction. Meanwhile, the total luminosity (and hence the central condensation) remains remarkably constant (Stothers 1966a; Stothers and Chin 1968). However, the depletion of hydrogen amounts to only a small change in $q_{s}$, so that the final value of $q_{s}$ for any mass will always be equal to or less than $\sim 0.60$ at the end of core helium burning. The subsequent evolution takes the star rapidly back into the region of red supergiants.

To complete our investigation of the stability of early-type supergiants, we have tested models for several of the masses at an earlier stage when the hydrogen shell is first set up after core hydrogen burning. Although the central condensation is not so severe at this stage, the "core contraction" models showed the same markedly negative result as the core helium-burning models.

The general conclusion to be drawn from our analysis is that all massive blue stars beyond the main-sequence phase of evolution should be stable against nuclear-energized 
pulsations. If pulsational instability disrupts stars on the main sequence above a certain critical mass, then the upper boundary of the H-R diagram should be delineated by the evolutionary track of a star of that critical mass.

\section{CRITICAL STELLAR MASS, CHEMICAL COMPOSITION, AND DISTANCE MODULUS OF THE MAGELLANIC CLOUDS}

\section{a) $B$ and $A$ Supergiants}

According to the evidence in the Magellanic Clouds, the domain of stars on the H-R diagram has a sharp upper bound. This is especially noticeable among the numerous blue supergiants of the Large Cloud (Feast, Thackeray, and Wesselink 1960). The upper bound nearly follows a line of constant bolometric magnitude, in agreement with the theoretical track for massive supergiants which are in the core helium-burning phase of evolution (Stothers 1966a; Stothers and Chin 1968).

It therefore appears likely that the luminosity function becomes discontinuous at some critical mass. By using an assumed distance modulus to the Magellanic Clouds, we may either find the critical mass and the hydrogen-helium abundance of the Cloud Population I, or by using a spectroscopically determined hydrogen-helium abundance, we may find the critical mass and the distance modulus. Thus we should be able to obtain a self-consistent set of values for all three parameters.

The procedure adopted is the following. First, we assume that the brightest supergiants have the critical mass for pulsational stability on the main sequence. (This assumption can be checked later through self-consistency of all the results.) Second, we adopt a fixed value for the difference in bolometric magnitude $\Delta M_{\text {bol }}$ between the brightest supergiants and their (unobserved) main-sequence progenitors. Thus we are assuming that this difference is independent of mass and chemical composition. To zero order, the assumption is valid. For example, the difference in $M_{\text {bol }}$ between the blue helium-burning models and the initial main-sequence models for various masses (with $\left.X_{e}=0.70, Z_{e}=0.03\right)$ is $\Delta M_{\mathrm{bol}}=-1.0(30 M \odot),-0.8(60 M \odot)$, and $-0.6(100 M \odot)$. The difference in $M_{\mathrm{bol}}$ for two different hydrogen-helium abundances (at $\sim 15 M \odot$ ) is $\Delta M_{\mathrm{bol}}=-1.30\left(X_{e}=0.70, Z_{e}=0.03\right)$ and $-1.35\left(X_{e}=0.90, Z_{e}=0.02\right)$. These zero-order variations are small when considering the uncertainties in the bolometric corrections which must be applied to the observational data. However, even to first order, our assumption above is good if we use the value of $\Delta M_{\mathrm{bol}}$ for the case of $60 M \odot, X_{e}=$ 0.70 , and $Z_{e}=0.03$ (which will turn out to be close to the inferred values for the Magellanic Clouds). Thus we adopt henceforth $\Delta M_{\mathrm{bol}}=-0.8$.

The third step in our procedure is to use (1) the theoretical $M-L-\left(X_{e}, Z_{e}\right)$ relation for the initial main sequence, which is expressed exactly (on the assumption of a purely electron-scattering opacity) in terms of three equations in the five parameters $A, C, M$, $L$, and $\left(X_{e}, Z_{e}\right)$; and (2) the theoretical $M-\left(X_{e}, Z_{e}\right)$ relation for the critically stable mass on the main sequence, which is expressed in terms of one equation relating $M$ and $\left(X_{e}, Z_{e}\right)$. These relations and the definitions of $A$ and $C$ are given in the Appendix. It is clear that by specifying $L$ (or $M_{\text {bol }}$ ) we may solve for the other four unknowns in this system of four equations, in particular for $M$ and $\left(X_{e}, Z_{e}\right)$. Or, by specifying $\left(X_{e}, Z_{e}\right)$, we may solve for $M$ and $M_{\mathrm{bol}}$. Results covering the whole range of $X_{e}$ are presented in Table 3 (assuming $Z_{e}=0.03$ ).

We turn now to consideration of the observational data for the brightest stars in the Magellanic Clouds. Feast, Thackeray, and Wesselink (1960) have tabulated $V$ magnitudes, $B-V$ colors, and MK spectral types and luminosity classes for most of these stars. The objects for which complete data are available in the spectral range B0-A5 are all supergiants of luminosity class $\mathrm{I}$. In addition, five bright $\mathrm{O}$ stars (three with complete data) and some $F, G$, and $\mathrm{K}$ supergiants are also listed. We have reduced the observed magnitudes by applying (1) extinction corrections based on the intrinsic colors of super- 
giants as tabulated by Johnson (1966) and on the customarily assumed ratio of total to selective absorption $A_{V} / E_{B-V}=3$ and (2) bolometric corrections for supergiants also tabulated by Johnson (1966). The H-R diagram in apparent bolometric magnitude is shown in Figure 2. The superimposed line corresponds to the limit of completeness $V=$ 10.6 estimated by Feast $e t$ al. for the B and A stars. The data for the stars of later type are incomplete, except for the brightest Cepheids.

According to Bok (1966) and others, the "best values" for the true distance modulus to the Magellanic Clouds are $(m-M)_{0}=18.7$ (Large Cloud) and $(m-M)_{0}=19.0$ (Small Cloud). These values are based primarily on the mean magnitudes of RR Lyrae variables found in Cloud clusters and a mean extinction correction of 0.3 mag. However, they agree with the values based on the apparent diameters of $\mathrm{H}$ II rings, which are independent of the extinction correction and presumably of uncertainties in quantities like stellar mass and chemical composition. Therefore, the adopted values of distance modulus are indebendent of the determinations below based on the structure and evolution of massive stars.

TABLE 3

Critical $M-L-\left(X_{e}, Z_{e}\right)$ Relation For PUlsational Stability ON THE INITIAL MAIN SEQUENCE*

\begin{tabular}{|c|c|c|c|c|c|}
\hline$M_{\mathrm{bol}}$ & $X_{e}$ & $M / M \odot$ & $M_{\mathrm{bol}}$ & $X_{e}$ & $M / M_{\odot}$ \\
\hline $\begin{array}{ll}-8 & 50 \\
-8 & 60 \\
-8 & 70 \\
-8 & 80 \\
-8 & 90 \\
-9 & 00 \\
-9 & 10\end{array}$ & $\begin{array}{rr}0 & 05 \\
10 \\
\\
\\
45 \\
20 \\
26 \\
\quad 32 \\
039\end{array}$ & $\begin{array}{l}14 \\
16 \\
18 \\
21 \\
24 \\
27 \\
31\end{array}$ & $\begin{array}{ll}-9 & 20 \\
-9 & 30 \\
-9 & 40 . \\
-9 & 50 \\
-9 & 60 \\
-9 & 70 \\
-9 & 73\end{array}$ & $\begin{array}{rr}0 & 46 \\
54 \\
62 \\
\\
72 \\
82 \\
\quad 93 \\
0 \quad 97\end{array}$ & $\begin{array}{l}36 \\
42 \\
49 \\
56 \\
65 \\
76 \\
80\end{array}$ \\
\hline
\end{tabular}

$* Z_{e}=003 ;$ critical $\log A=255$.

The chemical composition of Population I in the Clouds has recently been discussed by Bok (1966). There seems to be good evidence for a slight helium and metals deficiency with respect to comparable objects in our own Galaxy. Specifically, the iron group of elements in the visually brightest star in the Large Cloud, HD 33579, is roughly 50 per cent less abundant than in the comparable galactic supergiant a Cygni (Przybylski 1965). The abundances of helium and oxygen in the 30 Doradus Nebula (Large Cloud) and the abundance of helium in NGC 346 (Small Cloud) are about 30 per cent lower than in the Orion Nebula (Faulkner 1964). Adopting Faulkner's values for the $\mathrm{He} / \mathrm{H}$ ratio and a metals abundance of $Z_{e}=0.02-0.03$, we find $X_{e}=0.75$ for the hydrogen abundance of the young population in the Magellanic Clouds.

A comparison between the observational data and our theoretical models can now be made. First, we consider the brightest stars in the Large Cloud. Because of the uncertainties in the extinction corrections (colors) and bolometric corrections, we have averaged the apparent bolometric magnitudes of the first five brightest stars, yielding $m_{\mathrm{bol}}=8.4$ for the critical luminosity cutoff. With $(m-M)_{0}=18.7$ and $\Delta M_{\mathrm{bol}}=-0.8$, this corresponds to an absolute magnitude on the main sequence of $M_{\mathrm{bol}}=-9.5$. According to Table 3 , the critical mass and the hydrogen abundance are then $56 M \odot$ and $X_{e}=0.72$. Alternatively, with $X_{e}=0.75$ and $\Delta M_{\mathrm{bol}}=-0.8$, the critical mass and the distance modulus are $60 M \odot$ and $(m-M)_{0}=18.7$, respectively. The self-consistency of these results is remarkable in view of the uncertainties involved in both the observational data and the model assumptions. We conclude that (1) the upper luminosity bound of the supergiants probably reflects the maximum stable mass on the main sequence, (2) this mass occurs at about $60 M \odot,(3)$ the age-zero chemical composition is 
$X_{e}=0.75, Z_{e}=0.02-0.03$, and (4) the distance modulus to the Large Cloud is $(m-M)_{0}=18.7$.

In their theoretical discussion of the maximum stable mass of main-sequence stars, Schwarzschild and Härm (1959) showed that with the above chemical composition, stars in the range $60-65 M \odot$ would probably manage to avoid pulsational disruption. In this regard, it is interesting to note that the two brightest Cloud supergiants should have masses of $64 M \odot$, according to our results.

The data on the Small Cloud are more meager. With $(m-M)_{0}=19.0, \Delta M_{\mathrm{bol}}=$ -0.8 , and $X_{e}=0.75$, the brightest star apparently has a mass of $48 M \odot$. However, this is not inconsistent with the results for the Large Cloud because of the fewer statistics in the smaller galaxy. In the Large Cloud, nine stars have masses equal to or greater than $48 M \odot$, but the total mass ratio of the two Clouds is also eight or nine (Allen 1963).

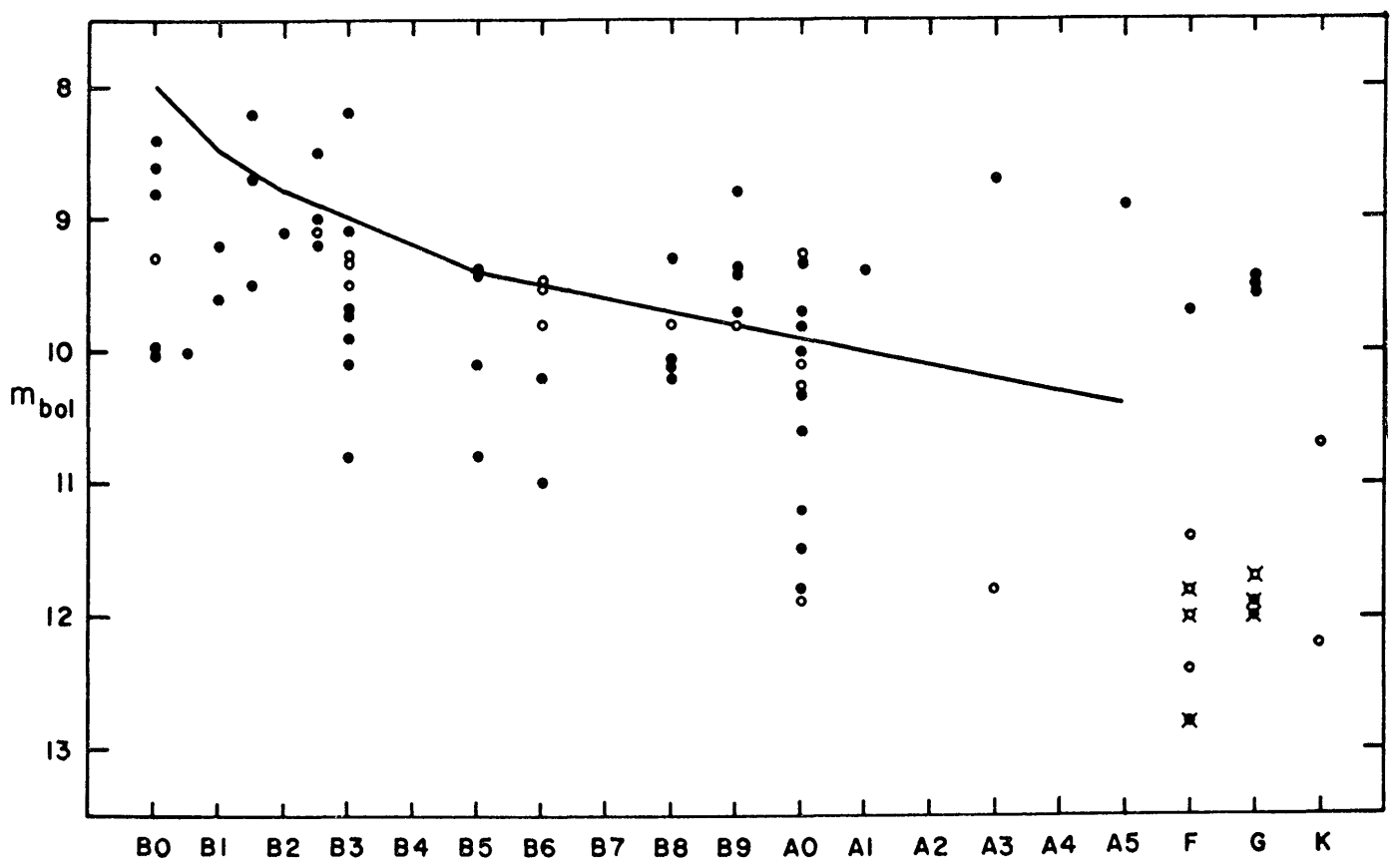

FIG. 2.-Observational H-R diagram in apparent bolometric magnitude for the brightest supergiants in the Large Magellanic Cloud (filled circles) and in the Small Magellanic Cloud (open circles). Crosses denote Cepheid variables. The line corresponds to the limit of completeness (Feast et al. 1960).

\section{b) Cepheids}

Another independent way of estimating (crudely) the critical mass utilizes the observed cutoff of Cepheids on the H-R diagram (Fig. 2). All we need to specify in this case is the chemical composition, which we may take as $X_{e}=0.75, Z_{e}=0.02-0.03$ from the spectroscopic results. Now, according to calculations by Hofmeister (1967) for $9 M \odot$ with $X_{e}=0.60, Z_{e}=0.04$ (which are sufficiently close to the adopted Cloud composition values for our present purpose), the third evolutionary passage of a star across the Cepheid strip during helium burning is the longest passage. It requires about 1 per cent of the helium-burning lifetime. Iben (1966) calculated the "third" passage for a star of $15 M \odot$ with $X_{e}=0.71, Z_{e}=0.02$ (which never went through the first and second passages because of his assumption about the internal mixing; cf. Stothers and Chin 1968). This passage is at least four times faster relative to the corresponding passage at $9 M \odot$. Moreover, the absolute helium-burning lifetime is a factor 2 shorter at $15 M \odot$ than at $9 M \odot$. Even if the birth-rate function of the brightest stars does not decrease 
with increasing mass, we may still judge from the admittedly incomplete statistics of the Large Cloud in Figure 2 that the Cepheid cutoff ought to occur at some mass below $15 M \odot$ (but not as low as $9 M \odot$ ). We shall simply adopt an average mass of $12 M \odot$. Now the brightest observed Cepheids lie $3.5 \mathrm{mag}$ (bolometric) below the upper bound of the supergiants. This implies, from our models, a mass of $\sim 55 M \odot$ for the upper bound. This is certainly satisfactory agreement with our previous, much more precise determination of the critical mass.

Using our previous determination, we find a "better" mass for the brightest Cepheids to be $13 M \odot$ (Large Cloud) and $15 M \odot$ (Small Cloud).

\section{c) O Stars}

A few $\mathrm{O}$ stars in the Magellanic Clouds appear to be even more luminous than the supergiants of critical mass. In particular, three early $\mathrm{O}$ stars for which reliable data are available occur at $M_{v}=-7.0$. Two are classified as Of stars, which are known to be brighter than ordinary $\mathrm{O}$ stars (Kopylov 1959). The bolometric corrections for such stars are very uncertain. If we assume that the five 05 stars in the $h$ and $\chi$ Persei association (Wildey 1964) are main-sequence objects characterized by an Orion-like chemical composition, $X_{e}=0.67, Z_{e}=0.03$ (Faulkner 1964), and by the corresponding critical mass $52 M \odot$ (Table 3), and if we adopt the distance modulus determined by Schild (1967), then the theoretical models predict a bolometric correction of -3.8 . Applying this bolometric correction to the three brightest $\mathrm{O}$ stars in the Clouds, we find masses of $115 \mathrm{M} \odot$ if the stars are main-sequence objects, or $75 M \odot$ if they are "supergiants" with O-type spectra. In either case, they are "superluminous" with respect to the critical mass. (If the stars are supergiants and actually cooler than indicated by their spectral types, the bolometric corrections must have been overestimated and the masses might be much smaller.)

If the stars are bright because of composition anomalies, their masses may be accordingly estimated. Let us imagine that they originally possessed the normal Cloud helium abundance, had evolved and then completely mixed at the end of hydrogen burning. Their new "initial" helium content is then much greater. Now hydrogen depletion has been calculated in the case of an original composition of $X_{e}=0.75, Z_{e}=0.03$ by Schwarzschild and Härm (1958) for several heavy masses. Interpolating in their results for the mean $X$ at core hydrogen exhaustion and using the formulae in the Appendix, we find masses of $68 M \odot$ and a new "initial" hydrogen content of $X_{e}=0.26$. Although such masses look reasonable, the critical stable mass for the new composition is only $24 M \odot$ (Table 3). No spectroscopic evidence exists that these O stars are hydrogen-deficient or violently unstable. Even if the stars were composed of pure helium, their masses would be $46 M \odot$, while the critical stable mass for pure-helium stars is only 7-8 $M \odot$ (Boury and Ledoux 1965). Any revision of our adopted bolometric correction for such "mainsequence" stars would almost certainly be toward a greater correction and would only increase the discrepancy between the inferred masses and the critical stable mass.

It is very likely that these O stars are actually optical multiples. Feast (1964) has evidence, for instance, that some of the bright "yellow" supergiants in the Small Cloud may be combinations of unresolved B and M supergiants. Many of Sharpless' (1954) multiple O-star systems in the Milky Way would appear unresolved at the distance of the Magellanic Clouds. If the three brightest Cloud O stars were optical triples, then each component might have a mass of $\sim 50 M \odot$ (assuming equal masses for the components).

This composite effect would probably not appear as frequently among the supergiants, if we assume, in analogy with observed associations in our own Galaxy, that the stars move away rapidly from their place of origin. Hence we are justified in treating the observed Cloud supergiants as primarily single stars. 


\section{CRITICAL STELLAR MASS IN OUR GALAXY}

\section{a) B Supergiants}

The evidence for the critical stellar mass in our own Galaxy is not as complete as in the Magellanic Clouds because of interstellar extinction. The most luminous object known in a galactic cluster is probably $\zeta^{1}$ Sco in NGC 6231. On the basis of the observational parameters Sp. $=$ B1.5 Ia $+, V=4.86, B-V=+0.46$ (Code and Houck 1958), and $(m-M)_{0}=11.6$ for NGC 6231 (Houck, quoted in Blaauw 1961), we may apply Johnson's (1966) intrinsic colors and bolometric corrections of supergiants to obtain $M_{\mathrm{bol}}=-10.4$. This corresponds to a mass of $58 M \odot$ for $X_{e}=0.67, Z_{e}=0.03$. A comparably luminous object is the highly reddened B5 supergiant, No. 12 in VI Cygni, for which Sharpless (1957) estimated roughly $M_{v}=-9.5$. This also corresponds to a mass of $58 M \odot$. Although the luminosities and hence masses of $\xi^{1}$ Sco and VI Cygni No. 12 are very uncertain, the masses are nevertheless not significantly in excess of the critical mass, which is $52 M \odot$ for the adopted (Orion-like) chemical composition.

\section{b) Trumpler O Stars}

Extremely large masses were found by Trumpler (1935) for several luminous O stars in young galactic clusters by interpreting the anomalous redshift in their spectra as a relativity effect. However, good evidence now exists that the redshift must arise, at least primarily, from other causes. We mention five major reasons for disbelieving such large masses. First, many of the Trumpler stars have spectra which resemble the spectra of other O stars with normal masses; Struve (1950) mentions S Mon in this connection. Second, two Trumpler stars are known to be spectroscopic binaries, for which the values of $M_{1} \sin ^{3} i$ look quite reasonable: $23 M \odot$ for $\mathrm{DH}$ Cep and $34 M \odot$ for $\tau \mathrm{CMa}$ (Struve 1950); Trumpler gives 75 and $300 M \odot$ for these stars, respectively. Third, the Trumpler stars show considerable scatter in the mass-luminosity plane (Chandrasekhar 1939), whereas a relation such as $L \sim M^{1}{ }^{5}$ would be expected theoretically (independently of evolutionary effects since stars of extremely high mass evolve at nearly constant luminosity). Fourth, on the basis of Trumpler's own luminosities (Chandrasekhar 1939), the brightest object, $\tau \mathrm{CMa}$, has a mass of only $\sim 60 \mathrm{M} \odot$ if we apply our theoretical models, making the assumptions that the chemical composition is Orion-like and that the star lies on the main sequence. Fifth and finally, the redshift in the spectrum may be due to dynamic motions (prominences) in the atmosphere, with inward streaming observed preferentially (Struve 1950).

\section{c) Of Stars}

Blaauw (1961) inferred luminosities for some of the "runaway" stars near stellar associations by integrating their orbits back to their presumed place of origin. According to our models, a few of Blaauw's runaway Of stars appear to be somewhat "superluminous" with respect to the critical mass, although not by so large an amount as in the case of the Magellanic Cloud $\mathrm{O}$ stars. While the runaway objects are almost certainly single stars, their luminosities are extremely uncertain. Consequently, we should not make inferences about the critical mass from them. Nevertheless, some of the Of stars in the neighborhood of the $h$ and $\chi$ Persei association (Wildey 1964) also seem to be rather "superluminous." Thus it is interesting that the emission-line intensities of Of stars are known to vary on a time scale of a few hours (Oke 1954), which is similar to the pulsational time scale of models of very massive hydrogen-burning stars (Schwarzschild and Härm 1959). However, the observed variations are by no means periodic. Moreover, if, in analogy with the other early-type emission-line stars, the Of stars are actually giants or supergiants (Kopylov 1959), then their masses are certainly below the critical mass. 


\section{d) Binaries}

A more intriguing result of Blaauw's (1961) work is his inference of exceedingly high masses (up to $1000 M \odot$ ) for the supposed original companions of the runaway starscompanions which dissipated their mass quickly in supernova explosions. Perhaps the dissipation of their mass began in pulsational instability on the main sequence. Apart from such indirect evidence for large masses in binary systems, the largest mass determined explicitly from a binary orbit is $\sim 60 M \odot$ for the secondary component of Plaskett's star, HD 47129 (Sahade 1962). Thus no observed binaries have components with masses exceeding substantially the critical mass.

\section{STABILITY OF THE B SUPERGIANTS}

From the data for the Magellanic Clouds it is apparent that a star of critical mass on the main sequence can evolve to the blue-supergiant region and remain there without disruption. Thus no violent displacements seem to take place, in agreement with our theoretical results.

Changes in any observed features are apparently small scale. For instance, nearly all the brightest blue supergiants in the Magellanic Clouds show emission lines (Feast et al. 1960). This is in accord with the observation in our own Galaxy that the strength of hydrogen emission increases with luminosity. The emission is usually $\mathrm{P}$ Cygni in character, suggesting an expanding shell. However, it is probably nearly constant with time, implying a steady outflow of matter (Abt, quoted in Weymann 1963; Thackeray 1964). Morton's (1966) preliminary estimate of the rate of mass loss from spectra taken of galactic $\mathrm{O}$ and $\mathrm{B}$ supergiants in the ultraviolet was greatly exaggerated, and the rate is

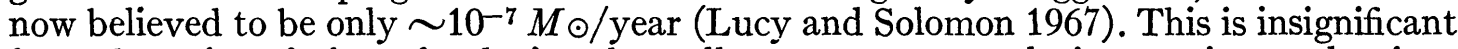
from the point of view of reducing the stellar mass on an evolutionary time scale, since helium burning is completed in less than a million years for a star of mass greater than $15 M \odot$.

The mechanism causing the mass loss is believed to be atmospheric radiation pressure, not pulsation. That this is the case has been demonstrated by Lucy and Solomon (1967) in a theoretical investigation of the dynamical atmosphere of such a star. The investigation of the present paper lends further support to the idea that the mechanism is not pulsation.

Abt (1957), however, attributed the small radial-velocity and visual-light changes in some late supergiants of types B-F to a pulsational origin. For these stars of type later than those with which we are primarily concerned in this paper, little can be said at present. Nevertheless, alternate hypotheses involving binaries and atmospheric motions have been proposed.

A sequence of photometric and spectroscopic studies with high time resolution is now available for some of the brightest B supergiants in the Magellanic Clouds. It appears that short-period changes in the emission-line profiles and intensities are non-periodic atmospheric effects (Thackeray 1964), probably leaving the bolometric magnitude constant. Although long-period changes in visual light have been observed in some stars, notably S Doradus, this star appears bluer at minimum than at maximum (Feast et al. 1960), suggesting that its bolometric magnitude may not be varying (or at least not drastically). Short-period variations in visual light among the brightest stars, if such variations exist at all, seem to average less than $0.01 \mathrm{mag}$. Bok (1966) concludes: "The impression one has is of remarkable constancy of brightness of these stars." Our theoretical calculations tend to support this view.

One of us (R. S.) has been supported by an NAS-NRC postdoctoral research associateship under the National Aeronautics and Space Administration. We would like to thank Dr. Leon Lucy for his interest in this problem and Dr. Robert Jastrow for his hospitality at the Institute for Space Studies. 


\section{APPENDIX}

\section{THE $M-L-(X, Z)$ RELATION FOR VERY MASSIVE STARS}

The basic equilibrium equations for massive stars, in which electron scattering is the only opacity source and nuclear-energy generation is confined to the convective core, may be cast into non-dimensional form by using the variables defined in Paper I (Stothers 1963). The equations for the envelope then contain only the parameters $A$ and $C$ :

$$
A=10^{-0}{ }^{096} \mu_{e}^{4}\left(\frac{M}{M_{\odot}}\right)^{2}, \quad C=10^{-5} 335 \frac{1+X_{e}}{\mu_{e}^{4}} \frac{L}{L_{\odot}}\left(\frac{M_{\odot}}{M}\right)^{3} .
$$

The equations for the convective core contain only the parameter $\beta_{c}$, the ratio of gas pressure to total pressure at the center. The chemical composition nowhere appears explicitly except in the variable $j=l(1+X) /\left(1+X_{e}\right)$. But $j$ may be related to $l=\mu / \mu_{e}$ by the approximation $j=$ $l^{2} 285$.

Once $A$ is specified, the equations may be solved for the two eigenvalues $C$ and $\beta_{c}$. An evolutionary stage is determined by also specifying $l_{c}$.

Integrations of these equations are available in the literature for a large number of $A$ values (e.g., Stothers 1963, 1965, 1966b). In the case of homogeneous stars, the relation between $C$ and $A$ may be represented by

$$
\log C=-3.618+0.509(\log A)-0.274(\log A)^{2}+0.0176(\log A)^{3}
$$

in the range $1.4 \leq \log A \leq 5.0$. For a standard composition given by $X_{e}=0.70$ and $Z_{e}=0.03$ $\left(\mu_{e}=0.618\right)$, the limits on $A$ correspond to 15 and $1000 M \odot$.

If two of the three parameters $M, L$, and $\left(X_{e}, Z_{e}\right)$ are given, then the third parameter may be determined from the above set of relations without performing new integrations. Strictly speaking, $\left(X_{e}, Z_{e}\right)$ is not a single parameter, but for $Z_{e}$ small, it may be treated as such since $Z_{e}$ appears only through $\mu_{e}=4 /\left(5 X_{e}-Z_{e}+3\right)$.

Two explicit uses of these relations will be pointed out here. (1) If the mode of semiconvection adopted for hydrogen-burning stars is that of Sakashita and Hayashi, then the semiconvective zone has a maximum growth in mass fraction at $\log A=2.6$. Both of the two convectively unstable regions in the star (semiconvective zone and convective core) have a combined maximum growth at $\log A=3.7$ (Stothers 1966b). For the standard composition, these $A$ values correspond to $\sim 60$ and $\sim 200 M \odot$, according to the definition of $A$. (2) If the linearized perturbation equations applicable to massive pulsating stars in the quasi-adiabatic approximation are cast into non-dimensional form, then the sign of the stability coefficient (indicating stability or instability) depends only on non-dimensional quantities and $\nu$, the temperature exponent in the nuclear-energy generation formula (neglecting the small contribution from surface running waves). For hydrogen-burning stars, $\nu$ is constant over a wide range of masses. With $\nu=13$, pulsational instability occurs for $\log A \geq 2.55$ (Schwarzschild and Härm 1959). For the standard composition, this corresponds to $M \geq 55 M \odot$.

\section{REFERENCES}

Abt, H. A 1947, Ap J., 126, 138.

Allen, C. W. 1963, Astrophysical Quantities (London: Athlone Press).

Blaauw, A 1961, B A.N., 15, 265.

Bok, B J. 1966, Ann. Rev Astr. and Ap, 4, 95.

Boury, A. 1963, Ann. d'ap, 26, 354.

Boury, A., and Ledoux, P. 1965, Ann. d'ap , 28, 353.

Chandrasekhar, S. 1939, An Introduction to the Study of Stellar Structure (Chicago: University of Chicago Press), p 490 .

Code, A. D , and Houck, T. E. 1958, Pub. A.S.P., 70, 261.

Cox, J. P. 1955, Ap. J., 122, 286. 
Faulkner, D. J. 1964, The Galaxy and the Magellanic Clouds (IAU/URSI Symp. No 20), ed. F. J Kerr and A. W. Rodgers (Canberra: Australian Academy of Science), p. 310.

Feast, M W. 1964, The Galaxy and the Magellanic Clouds (IAU/URSI Symp. No. 20), ed F. J Kerr and A. W. Rodgers (Canberra: Australian Academy of Science), p. 330

Feast, M W., Thackeray, A. D., and Wesselink, A. J. 1960, M.N R.A.S., 121, 337.

Hayashi, C., and Cameron, R. C. 1962, Ap.J., 136, 166.

Hofmeister, E. 1967, Zs.f. Ap., 65, 164.

Iben, I , Jr. 1966, $A$ p. J., 143, 516.

Johnson, H. L. 1966, Ann. Rev Astr. and Ap, 4, 193.

Kopylov, I. M. 1959, Ann. d'ap. Suppl., 8, 41.

Ledoux, P. 1941, Ap J., 94, 537.

Lucy, L. B , and Solomon, P. M. 1967, A.J., 72, 310

Morton, D. C. 1966, A.J., 71, 172.

Oke, J. B. 1954, Ap.J., 120, 22.

Przybylski, A. 1965, Nature, 205, 163.

Sahade, J , ed. 1962, Symposium on Stellar Evolution (La Plata: National University of La Plata), p 185.

Sakashita, S., Ôno, Y., and Hayashi, C. 1959. Progr. Theoret. Phys. (Kyoto), 22, 830.

Schild, R. 1967, Ap.J., 148, 449.

Schwarzschild, M., and Härm, R. 1958, Ap.J., 128, 348

. 1959, ibid., 129, 637.

Sharpless, S. 1954, $A$ p.J., 119, 334

Stother. 1957, Pub. A.S.P., 69, 239.

Stothers, R. 1963, Ap.J., 138, 1074.

$$
\begin{aligned}
& \text {. 1964, ibid., 140, } 510 \\
& \text { 1965, ibid., 141, } 671 \text {. }
\end{aligned}
$$$$
1966 a \text {, ibid., 143, } 91 \text {. }
$$

. $1966 a$, ibid., 143, 91.

Stothers, R., and Chin, C.-w. 1968, Ap.J., 152, 225.

Struve, O 1950, Stellar Evolution (Princeton, N J.: Princeton University Press).

Thackeray, A. D. 1964, The Galaxy and the Magellanic Clouds (IAU/URSI Symp No 20), ed. F. J. Kerr and A. W. Rodgers (Canberra: Australian Academy of Science), p. 370.

Trumpler, R. J. 1935, Pub. A.S.P., 47, 249.

Weymann, R. 1963, Ann. Rev. Astr. and Ap., 1, 113

Wildey, R. L. 1964, Ap. J. Suppl, 8, 439.

Copyright 1968 The University of Chicago Printed in U S A 